\title{
Avaliação clínico-laboratorial de bovinos Nelore infectados experimentalmente com Trypanosoma vivax ${ }^{1}$
}

\author{
Maria A. M. Schenk ${ }^{2}$, Carla L. Mendonça ${ }^{3}$, Cláudio R. Madruga², Aguemi \\ Kohayagawa $^{4}$ e Flábio R. Araújo ${ }^{2}$
}

\begin{abstract}
Schenk M.A.M., Mendonça C.L., Madruga C.R., Kohayagawa A. \& Araújo F.R. 2001. [Clinical and laboratorial evaluation of Nellore cattle experimentally infected with Trypanosoma vivax] Avaliação clínico and laboratorial de bovinos nelore infectados experimentalmente com Trypanosoma vivax. Pesquisa Veterinária Brasileira 21(4):157-161. Embrapa Gado de Corte, Cx. Postal 154, Campo Grande, MS 79002-970, Brazil.

In order to evaluate the clinical-laboratorial alterations, six Nellore calves were inoculated with $10^{7}$ Trypanosoma vivax isolated from Poconé region, Mato Grosso, Brazil. The animals were evaluated daily for rectal temperature, packed cell volume (PCV), parasitemia, antibody production, color of mucous membranes, behavior and appetite. Blood and serum samples for biochemical evaluation for aspartate aminotransferase (AST), alkaline phosphatase (AF), gamma glutamyltransferase (GGT), cholesterol, urea, creatinine, creatine kinase (CK), calcium, phosphorus and proteinogram were collected on days 4, 8, 12, 16, 23 and 30 post inoculation (DPI). During the following 6 months rectal temperature, PCV and parasitemia were evaluated weekly. T. vivax was evidenced from $1 \mathrm{DPI}$ in all calves and persisted until day 30 in five of six animals. A remarkable decrease $(\mathrm{p}<0.05)$ of PCV mean value $(25 \%)$ was observed on 10 DPI. The animals presented no alterations in their clinical or serum biochemical state during the trial. Seroconversion took place 6 and $8 \mathrm{DPI}$, and all the animals remained seropositive during the 30 days of experiment. In all the experimental animals the occurrence of $T$. vivax infection was verified, characterized by the increase of corporal temperature, presence of the blood protozoa and reduction of the globular volume, without alterations in the other variables analyzed. Nellore calves, when experimentally inoculated with $T$. vivax, are able to establish a balance between host-parasite relationship.
\end{abstract}

INDEX TERMS: Trypanosoma vivax, calves, hematology, serum biochemistry, proteinogram.

RESUMO.- Avaliaram-se as alterações clínico-laboratoriais de seis bezerros Nelore, de ambos os sexos, inoculados experimentalmente com $10^{7}$ organismos viáveis de Trypanosoma vivax, isolados de bovinos da região de Poconé, Estado de Mato Grosso. Os animais foram observados diariamente, durante 30 dias, quanto aos parâmetros de temperatura retal, volume globular (VG), parasitemia, produção de anticorpos, coloração de mucosas, comportamento e apetite. Determi-

\footnotetext{
${ }^{1}$ Aceito para publicação em 20 de outubro de 2001.

${ }^{2}$ Embrapa Gado de Corte, Rodovia BR 262 km 4, Cx. Postal. 154, Campo Grande, MS 79002-970.

${ }^{3}$ Clínica de Bovinos, Campus Garanhuns, UFRPE, Cx. Postal 152, Garanhuns, PE 55290-000.

${ }^{4}$ Depto de Clínica Veterinária, FMVZ, Unesp, Campus de Botucatu, Cx. Postal 560, Botucatu, SP 18618-000.
}

naram-se os níveis séricos de aspartato aminotransferase (AST), fosfatase alcalina (FA), gama glutamiltransferase (GGT), creatina kinase (CK), colesterol, uréia, creatinina, cálcio, fósforo e o perfil eletroforético das proteínas séricas aos 4,8 , $12,16,23$ e 30 dias pós-inoculação (DPI). Durante os 6 meses seguintes, os animais foram observados semanalmente, avaliando-se a temperatura retal, o VG e a parasitemia. T. vivax foi evidenciado a partir do terceiro e quarto DPI em todos os bezerros e persistiu até o $30^{\circ}$ DPI em cinco dos seis animais em estudo. Ocorreu um decréscimo significativo $(\mathrm{p}<0,05)$ do valor médio do VG (25\%) aos dez DPI. Os animais não apresentaram qualquer alteração no quadro clínico, bem como na avaliação da bioquímica sérica durante o período experimental. A soroconversão ocorreu aos 6 e 8 DPI, permanecendo todos os animais soropositivos nos 30 dias experimentais. Bovinos nelores jovens, infectados experimentalmente 
com T. vivax, foram capazes de estabelecer um equilíbrio na relação hospedeiro-parasita.

TERMOS DE INDEXAÇÃO: Trypanosoma vivax, bezerros, hematologia, bioquímica sérica, proteinograma.

\section{INTRODUÇÃO}

A infecção por Trypanosoma vivax é responsável por perdas econômicas à bovinocultura de áreas tropicais, como África, Ásia, América Central e América do Sul, podendo levar à diminuição da produção, infertilidade, aborto, retardo no crescimento e mortalidade (Sekoni et al. 1990a, Okech et al. 1996a,b, Silva \& Davila 1998). Este agente é transmitido ciclicamente no continente africano pelas moscas do gênero Glossina e mecanicamente nas Américas e nas áreas da África onde não ocorre Glossina, por outros dípteros hematófagos (Levine 1973). Apesar de $T$. vivax ter sido introduzido na América do Sul no século XIX; no Brasil foi diagnosticado somente na década de 1970 , em búfalos no Estado do Pará (Shaw \& Lainson 1972). Desde então, esse parasito estava restrito ao norte do País, quando em 1995 foi detectado em bovinos na região de Poconé, Pantanal do Estado de Mato Grosso (Silva et al. 1996), e no Estado de Mato Grosso do Sul, nos municípios de Miranda (Paiva et al. 1997) e Aquidauana (Barbosa Jr et al. 2001).

$O$ agente desencadeia uma anemia hemolítica nos estágios iniciais da infecção, que é atribuída a mecanismos imunomediados. Os principais achados clínicos são febre, anemia, fraqueza progressiva, emaciação, aborto e síndromes hemorrágicas, que podem levar o animal à morte (Gaunt 2000). Entretanto, o efeito da infecção por T. vivax varia com o hospedeiro, o qual pode estabelecer um equilíbrio com o parasito e permanecer clinicamente normal, por um longo período (Radostits et al. 2000). No continente africano, algumas raças de bovinos são resistentes a $T$. vivax. Este fenômeno, denominado tripanotolerância, tem origem genética e ambiental, e pode variar ainda de acordo com a idade, estado nutricional, condições de estresse, infecções intercorrentes e cepas envolvidas (Uzoigwe 1986, Sekoni et al. 1990b, Leperre \& Claxton 1994, Mwangi et al. 1998, Van den Bossche \& Rowlands 2001).

No Brasil, são escassos os estudos sobre a patogenicidade da tripanossomose bovina. Esse trabalho teve por objetivo avaliar as alterações clínico-laboratoriais determinadas por T. vivax em bovinos Nelore infectados experimentalmente.

\section{MATERIAL E MÉTODOS}

O experimento foi realizado nas instalações do Laboratório de Sanidade Animal da Embrapa Gado de Corte, Campo Grande, Mato Grosso do Sul, e no Laboratório Clínico da Faculdade de Medicina, Veterinária e Zootecnia (FMVZ), Unesp-Campus de Botucatu, São Paulo. Utilizaram-se seis bovinos da raça Nelore Bos indicus), sorologicamente negativos para $T$. vivax, com aproximadamente 7 meses de idade, que foram mantidos em área de isolamento em prédio telado, livre de insetos. A dieta desses animais foi constituída de concentrado, feno de Brachiaria decumbens, sal mineral e água ad libitum.

Os animais foram inoculados por via intravenosa com o sangue colhido de um bezerro esplenectomizado, utilizado como doador, contendo $10^{7}$ organismos viáveis de $T$. vivax por $\mathrm{ml}$. A cepa empregada foi previamente isolada de bovinos da região do Pantanal de Poconé, Mato Grosso, e mantida criopreservada em nitrogênio líquido no Banco de Hemoparasitos da Embrapa Gado de Corte. A obtenção dos valores médios referentes ao momento controle de cada variável estudada foi resultante de três colheitas realizadas antes da inoculação, com intervalos de 48 horas. As variáveis, volume globular (VG), parasitemia, temperatura retal, coloração das mucosas, comportamento e apetite foram avaliadas diariamente durante os 30 dias experimentais e posteriormente, semanalmente, durante 6 meses. As amostras para determinação dos valores séricos de aspartato aminotransferase (AST), fosfatase alcalina (FA), gama glutamiltransferase (GGT), creatina kinase (CK), colesterol, uréia, creatinina, cálcio, fósforo e o perfil eletroforético das proteínas séricas foram colhidas aos $4,8,12$, 16,23 , e 30 dias pós-inoculação (DPI), estabelecendo-se sete momentos experimentais.

As amostras de sangue para a determinação do VG foram colhidas por venopunção da jugular, em anticoagulante ácido etilenodiaminotetracético (EDTA) a $10 \%$ e em tubos estéreis a vácuo, sem anticoagulante, para obtenção do soro, a partir do qual foram realizadas as determinações bioquímicas, o perfil eletroforético das proteínas séricas e o teste de imunoadsorção enzimática indireto (ELISA) para deteç̧ão de anticorpos contra T. vivax. Foram confeccionados esfregaços sangüíneos delgados para deteç̧ão de $T$. vivax, mediante a punção de capilares da margem da orelha, corados pela coloração rápida do tipo panótico (Hematocor-Biocolor). Para a determinação do VG, utilizou-se a técnica do microhematócrito (Coles 1984) e as provas bioquímicas foram realizadas em aparelho automatizado (Technicon RA-XT). A separação das frações protéicas foi efetuada segundo a técnica de eletroforese em gel de agarose, de acordo com o kit comercial (Celmgel-Celm) e a leitura efetuada por densitometria (DS35-Celm) em $520 \eta \mathrm{m}$, seguindo a metodologia descrita por Canavessi (1997). As amostras para o teste de ELISA foram colhidas, diariamente, durante os 30 dias experimentais, empregando-se a metodologia descrita por Madruga et al. (1999).

Procedeu-se a análise estatística dos dados pelo teste " $t$ " pareado, para amostras dependentes, comparando-se os resultados encontrados no momento controle com os momentos seguintes à inoculação (Curi 1997).

\section{RESULTADOS E DISCUSSÃO}

Os animais não desenvolveram o quadro clínico da tripanossomose, permanecendo todo o período de observação em bom estado nutricional, sem alteração do comportamento e do apetite. Não foram evidenciadas manifestações clínicas da enfermidade conforme as descritas por Silva et al. (1999), Gaunt (2000) e Radostits et al. (2000), sendo detectadas apenas algumas alterações laboratoriais, caracterizando uma forma subclínica de infeç̧ão, conforme também relatado por Otte et al. (1994) em bezerros infectados naturalmente por $T$. vivax na Colômbia.

Em rebanhos bovinos infectados por T. vivax no Pantanal Sul-Matogrossense, o agente contribui na condição de oportunista, pois as práticas de manejo adotadas, a movimentação freqüiente dos animais e o período de restrição alimentar prolongado, possibilitam a somatória de fatores que se traduzem no quadro clínico observado (Paiva et al. 1998). Para esses autores, a adaptação à transmissão mecânica reduz a variação antigênica do parasito, devendo este ser o principal 
fator a ser considerado quando da diferenciação das manifestações clínicas entre cepas africanas e americanas.

Observou-se uma elevação gradativa da temperatura retal a partir do $1^{\circ} \mathrm{DPI}$, alcançando valor máximo no $5^{\circ} \mathrm{DPI}\left(39,7^{\circ} \mathrm{C}\right)$, quando comparado ao momento controle, retornando aos valores pré-estabelecidos no decorrer do experimento (Fig. 1). Segundo Stöber (1993), as temperaturas corporais situadas entre os limites superiores ao normal e $40^{\circ} \mathrm{C}$ são denominadas de febre branda.

Para Radostits et al. (2000), o parasita é detectado mais

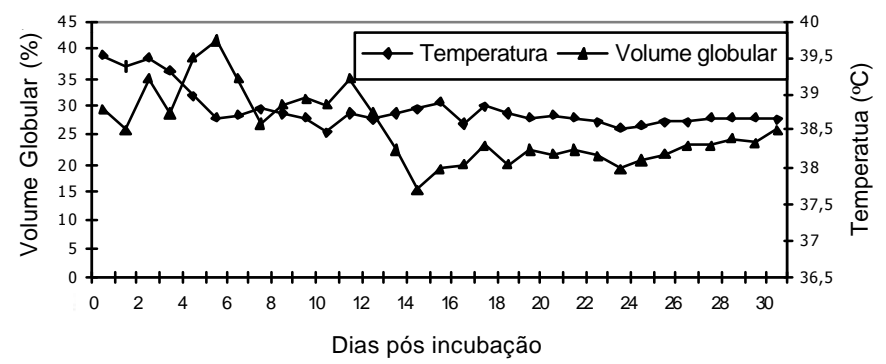

Fig. 1. Valores médios da temperatura retal e do volume globular de bovinos Nelore infectados experimentalmente com Trypanosoma vivax, isolado, Poconé, MT.

facilmente nos estágios iniciais da doença, quando o pico de parasitemia corresponde ao pico febril. A presença de $T$. vivax na circulação sanguínea foi observada a partir do $3^{\circ} \mathrm{DPI}$, em todos os bezerros, persistindo até o $30^{\circ} \mathrm{DPI}$ em cinco dos seis animais estudados. Resultados semelhantes foram relatados por Uzoigwe (1986), em bezerros zebus de 6 a 12 meses de idade, nos quais a tripanossomose não evoluiu de forma severa, sendo detectados baixos níveis de parasitemia e uma forma branda de anemia e, por Igbokwe et al. (1996), que verificaram a presença do agente aos dois DPI.

$\mathrm{O}$ valor médio do VG no momento controle, ou seja, antes da inoculação, foi de $39 \%$, havendo um decréscimo significativo $(\mathrm{p}<0,05)$ desta variável, que atingiu um valor mínimo de $25 \%$ aos $10 \mathrm{DPI}$, representando uma diminuição de 36\% (Fig. 1). Durante os 30 dias experimentais, os valores do VG não retornaram aos valores observados antes da inoculação.

Na avaliação semanal, realizada no decorrer de 6 meses, os animais mantiveram um quadro clínico estável, sendo a presença de T. vivax evidenciada em um dos animais com diminuição paralela do VG. Para Mwangi et al. (1998), a tripanotolerância está associada à capacidade do hospedeiro, uma vez infectado, em resistir ao desenvolvimento da anemia e controlar a parasitemia.

Após infecção experimental de bovinos por T. vivax, Sekoni et al. (1990b) evidenciaram uma leve e transitória diminuição do VG. A elevação da temperatura e o decréscimo dos valores do VG coincidiram com o aparecimento do parasito na corrente sangüínea, corroborando os achados de Saror (1979).

Embora os resultados tenham mostrado alterações signi-

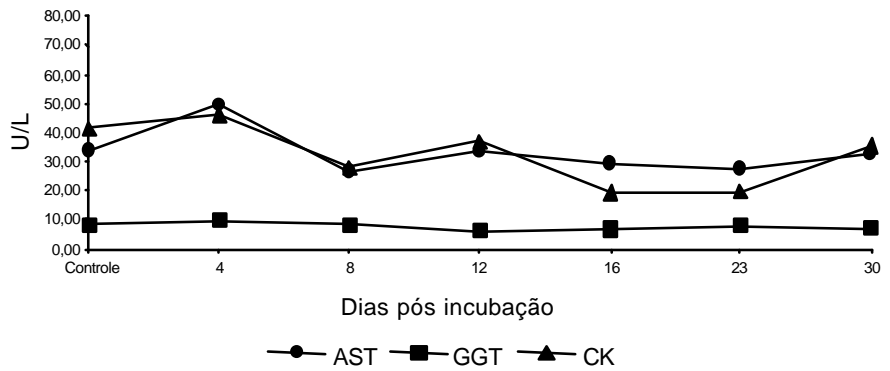

Fig. 2. Valores médios dos níveis de aspartato aminotransferase (AST), gama glutamiltransferase (GGT) e creatina kinase (CK) de bovinos Nelores infectados experimentalmente com Trypanosoma vivax, isolado, Poconé, MT.

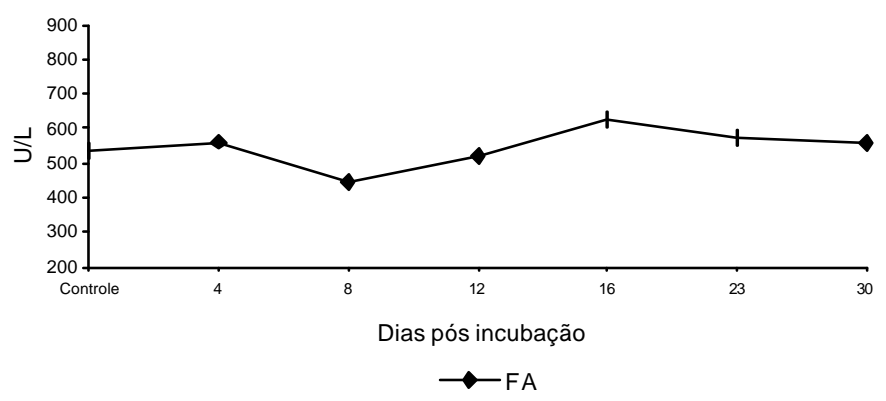

Fig. 3. Valores médios dos níveis de fosfatase alcalina (FA) de bovinos Nelore infectados experimentalmente com Trypanosoma vivax, isolado, Poconé, MT.

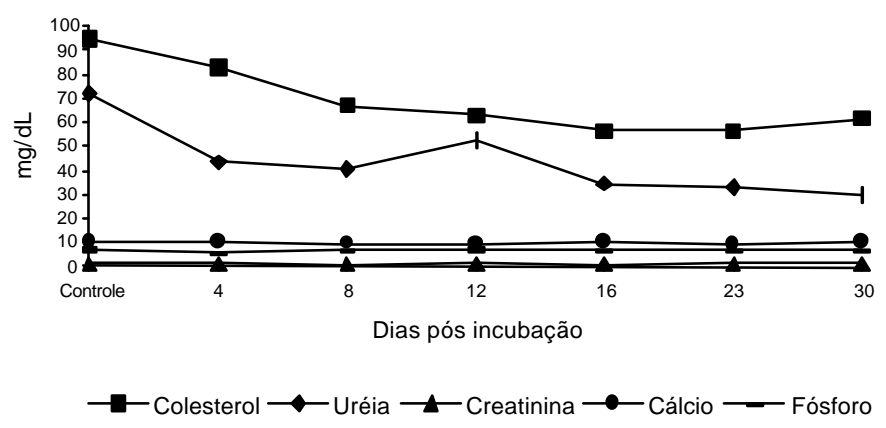

Fig. 4. Valores médios dos níveis de colesterol, uréia, creatinina, cálcio e fósforo $(\mathrm{mg} / \mathrm{dL})$ de bovinos Nelore infectados experimentalmente com Trypanosoma vivax, isolado, Poconé, MT.

ficativas $(\mathrm{p}<0,05)$, os valores de AST, GGT, CK (Fig. 2), uréia, creatinina, cálcio e fósforo (Fig. 4) permaneceram dentro de uma faixa de normalidade para a espécie bovina (Barros Filho 1995, Kaneko 1997, Morais et al. 2000a,b), não sendo detectadas alterações que evidenciassem uma disfunção hepática e renal. Em alguns momentos, os valores médios da FA (Fig. 3) foram superiores ao citado por Kaneko (1997), provavelmente devido à idade dos animais em estudo e, não por alteração hepática, pois não foram acompanhados pelo aumento da GGT (Fig. 2).

Um decréscimo significativo $(\mathrm{p}<0,05)$ foi constatado nos 


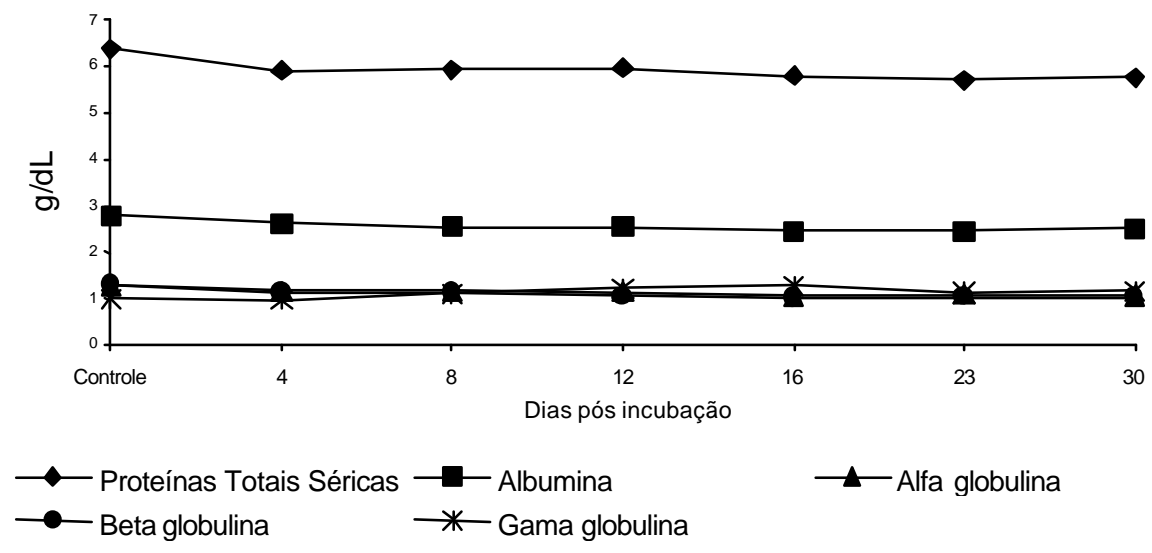

Fig. 5. Valores médios do perfil eletroforético das proteínas séricas $(\mathrm{mg} / \mathrm{dL})$ de bovinos Nelore infectados experimentalmente com Trypanosoma vivax, isolado, Poconé, MT.

níveis do colesterol (Fig. 4), que situaram-se abaixo do descrito por Kaneko (1997). Achados semelhantes foram encontrados por Katunga-Rwakishaya (1997), que trabalhando com ovinos infectados experimentalmente com Trypanosoma congolense, observaram que o declínio dos valores de colesterol inicia com o aparecimento dos tripanossomas na circulação, o que provavelmente esteja associado à liberação de produtos pelo parasito quando lisados.

Quanto ao proteinograma (Fig. 5), observou-se uma diminuição significativa $(\mathrm{p}<0,05)$ nos valores das proteínas totais séricas e da albumina a partir do $4^{\circ}$ e $8^{\circ} \mathrm{DPI}$, respectivamente, e um aumento significativo nos valores da gama globulina, a partir do $12^{\circ} \mathrm{DPI}$, provavelmente relacionado ao estímulo antigênico desencadeado pelo parasito. Katunga-Rwakishaya (1996) também observou uma diminuição nos níveis de albumina e uma hiperglobulinemia, sendo esta última atribuída a um aumento de IgG e principalmente IgM. Sekoni et al. (1990b) observaram um discreto decréscimo nos valores das proteínas plasmáticas totais de touros infectados experimentalmente com $T$. vivax, que atingiu valores mínimos na segunda semana pós-infecção.

No presente estudo, em todos os bezerros a soroconversão ocorreu entre o $6^{\circ}$ e $8^{\circ} \mathrm{DPI}$, permanecendo soropositivos durante os $\mathbf{3 0}$ dias de acompanhamento.

A infecção dos animais foi caracterizada por febre branda, pela presença do parasito no sangue, pela redução do VG e pela detecção de anticorpos contra T. vivax, ratificando os achados de Uzoigwe (1986) e Paiva et al. (1998), que relataram que a infecção pode ser controlada pelo animal, por meio da resposta imunológica, determinando a autocura. Acredita-se que o bom estado nutricional dos animais experimentais tenha sido um dos principais fatores dessa resposta benigna, dificultando a instalação de infecções intercorrentes e até mesmo uma forma mais grave da tripanossomose.

De acordo com os resultados, pode-se concluir que bovinos Nelore jovens em bom estado nutricional, infectados experimentalmente com $T$. vivax, são capazes de estabelecer um equilíbrio na relação hospedeiro-parasito.

\section{REFERÊNCIAS}

Barbosa Jr N.S., Madruga C.R., Osório A.L.A.R., Ribeiro L.R.R. \& Almeida R.F.C. 2001. Descrição de surto de tripanossomose bovina porTrypanosoma vivax, com morte perinatal no Pantanal de Aquidauana, MS. IV Congresso Brasileiro de Buiatria, Campo Grande, MS, p. 135.

Barros Filho I.R. 1995. Contribuição ao estudo da bioquímica clínica em zebuínos da raça Nelore (Bos indicus, Linnaeus 1758) criados no Estado de São Paulo. Dissertação de Mestrado, FMVZ-USP, São Paulo. 133p.

Canavessi A.M.O. 1997. Valores do perfil eletroforético das proteínas séricas de bovinos da raça Nelore (Bos indicus) criados na região de Botucatu, São Paulo: Influência dos fatores etários e sexuais. Dissertação de Mestrado, FMVZ, Unesp-Botucatu. 108p.

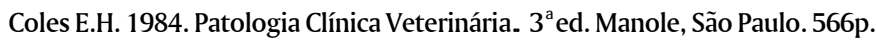

Curi P.R. 1997. Metodologia e Análise da Pesquisa em Ciências Biológicas. Tipomic, Botucatu. 263p.

Gaunt S.D. 2000. Hemolytic anemias caused by blood rickettsial agents and protozoa, p.154-162. In: Feldman B.F., Zinkl J.G. \& Jain N.C. (ed.) Schalm's Veterinary Hematology. 5th ed. Lippincott Williams \& Wilkins, Philadelphia.

Igbokwe I.O., Uman I.A., Omage J.J., Ibrahim N.D.G., Kadima K.B., Obagaiye O.K., Saror D.I. \& Esievo K.A.N. 1996. Effect of acute Trypanosoma vivax infection on cattle erythrocyte glutathione and susceptibility to in vitro peroxidation. Vet. Parasitol. 63:215-224.

Kaneko J.J. 1997 Clinical Biochemistry of Domestic Animals. 5th ed. Academic Press, San Diego. 932p.

Katunga-Rwakishaya E. 1996. Influence of Trypanosoma congolense infection on some blood inorganic and protein constituents in sheep. Rev. Élev. Méd. Vét. Pays Trop. 49(4):311-314.

Katunga-Rwakishaya E. 1997. The influence of dietary protein on some blood biochemical parameters in Scottish Blackface sheep experimentally infected with Trypanosoma congolense. Vet. Parasitol. 68:227-240.

Leperre P. \& Claxton J.R. 1994. Comparative study of trypanosomosis in zebu and N'Dama cattle in the Gambia. Trop. Anim. Hlth Prod. 26:139-145.

Levine, N.D. 1973. Protozoan Parasites of Domestic Animals and of Man. 2nd ed. Burgess Publ. Co., Minneapolis. 406p.

Madruga C.R., Araúijo F.R., Cruz T.M. \& Schenk M.A.M. 1999. Desenvolvimento de uma prova de imunoadsorção enzimática para detecção de anticorpos contra Trypanosoma vivax em bovinos: resultados preliminares. Embrapa-CNPGC, Campo Grande. 7p. (Pesquisa em Andamento 50, Embrapa-CNPGC)

Morais M.G., Gonçalves L.C., Lopes H.O.S., Costa M.F.V. \& Nunes A.B. 2000a. Variação sazonal de eletrólitos no sangue de vacas aneloradas sob pastejo contínuo de Brachiaria decumbens. Arq. Bras. Med. Vet. Zootec. 52(2):105110. 
Morais M.G., Rangel J.M., Madureira J.S. \& Silveira A.C. 2000b. Variação sazonal da bioquímica clínica de vacas aneloradas sob pastejo contínuo de Brachiaria decumbens. Arq. Bras. Med. Vet. Zootec. 52(2):98-104.

Mwangi E.K., Stevenson P., Gettinby G., Reid S.W.J. \& Murray M. 1998. Susceptibility to trypanosomosis of three Bos indicus cattle breeds in areas of differing tsetse fly challenge. Vet. Parasitol. 79:1-17.

Okech G., Dolan R.B., Stevenson P., Alushula H., Watson E.D., Luckins A.G. \& Omuse J.K. 1996a. The effect of trypanosomosis on pregnancy in trypanotolerant Orma Boran cattle. Theriogenology 46(3):441-447.

Okech G., Watson E.D., Luckins A.G. \& Makawiti D.W. 1996b. The effect of Trypanosoma vivax infection on late pregnancy and postpartum return to cyclicity in Boran cattle. Theriogenology 46(5):859-869.

Otte M.J., Abuabara J.Y. \& Wells E.A. 1994. Trypanosoma vivax in Colombia: epidemiology and production losses. Trop. Anim. Hlth Prod. 26:146-156.

Paiva F., Lemos R.A.A., Oshiro E.T., Salvador S.C. \& Nakazato L. 1997. Ocorrência de Trypanosoma vivax em bovinos no Estado de Mato Grosso do Sul. Revta Bras. Parasitol. Vet. 6(2)Supl.1:349.

Paiva F., Lemos R.A.A., Nakazato L., Mori A.E., Brum K.B. \& Bernardo K.C. 1998. Acompanhamento clínico, laboratorial e anatomopatológico de rebanho infectado por Trypanosoma vivax no Pantanal Sul-Matogrossense. Relatório. UFMS, Campo Grande, MS. 10p.

Radostits O.M., Gay, C.C, Blood D.C. \& Hinchcliff K.W. 2000. Veterinary Medicine. 9th ed. W.B. Saunders, London. 1877p.

Saror D.I. 1979. Classification of the anaemia of bovine trypanosomiasis. Vet. Rec. 105(4):96-98.

Sekoni V.O., Njoku C.O., Kumi-Diaka J. \& Saror D.I. 1990a. Pathological changes in male genitalia of cattle infected with Trypanosoma vivax and Trypanosoma congolense. Brit. Vet.J. 146(2):175-180.

Sekoni V.O., Saror D.I., Njoku C.O., Kumi-Diaka J. \& Opaluwa G.I. 1990b. Comparative haematological changes following Trypanosoma vivax and $T$. congolense infections in Zebu bulls. Vet. Parasitol. 35:11-19.

Shaw J.J. \& Lainson R. 1972. Trypanosoma vivax in Brazil. Ann. Trop. Med. Parasitol. 66(1):25-33.

Silva R.A.M.S., Silva J.A., Schneider R.C., Freitas J., Mesquita D., Mesquita T., Ramirez L., Dávila A.M.R. \& Pereira M.E.B. 1996. Outbreak of trypanosomiasis due to Trypanosoma vivax (Ziemann, 1905) in bovines of the Pantanal, Brazil. Mem. Inst. Oswaldo Cruz 91(5):561-562.

Silva, R.A.M.S. \& Davila, A.M.R. 1998. Trypanosoma vivax: biologia, diagnóstico e controle, p.123-145 In: Kessler R.H. \& Schenk M.A.M. (ed.) Carrapato, Tristeza Parasitária e Tripanossomose dos Bovinos. Embrapa Gado de Corte, Campo Grande, MS.

Silva, R.A.M.S. Ramirez L., Souza S.S., Ortiz A.G., Pereira S.R. \& Dávila A.M.R. 1999. Hematology of natural bovine trypanosomosis in the Brazilian Pantanal and Bolivian Wetlands. Vet. Parasitol. 85(1):87-93.

Stöber M. 1993. Identificação, anamnese, regras básicas da técnica de exame clínico geral, p.44-80. In: Dirksen G., Gründer H.D. \& Stöber M. (ed.) Rosenberger, Exame Clínico dos Bovinos. $3^{\underline{a}}$ ed. Guanabara Koogan, Rio de Janeiro.

Uzoigwe N.R. 1986. Self-cure in zebu calves experimentally infected with Trypanosoma vivax. Vet. Parasitol. 22:141-146.

Van den Bossche P. \& Rowlands G.J. 2001. The relationship between the parasitological prevalence of trypanosomal infections in cattle and herd average packed cell volume. Acta Tropica 78:163-170. 